\title{
A New Algorithm and a New Heuristic for Serial Supply Systems.
}

\author{
Guillermo Gallego* \\ Department of Industrial Engineering and Operations Research \\ Columbia University ${ }^{\dagger}$ \\ Özalp Özer \\ Department of Management Science and Engineering \\ Stanford University
}

September 1, 2004

\begin{abstract}
We present a new dynamic programming formulation for the stochastic multi-stage serial inventory system based on the cost of sub-system with fewer stages. A heuristic based on judiciously selected common downstream holding costs requires solving one newsvendor problem per stage. A closed form approximate upper bound allows for accurate sensitivity analysis. ${ }^{1}$
\end{abstract}

Key words: stochastic inventory systems; multi-echelon; newsvendor bound

*Corresponding Author: 326 Mudd Building, Columbia University, New York, NY gmg2@columbia.edu. ${ }^{\dagger}$ Research Supported by NSF Grant DMI-02-18104

${ }^{1}$ Acknowledgments: We wish to thank Haengju Lee, Ozge Sahin and Jay Sethuraman for useful suggestions on earlier versions of this note. 


\section{Series Systems}

The study of multi-stage serial inventory systems is central to the study of supply chain management both as a benchmark and as a building block for more complex supply networks. Existing policy evaluation and optimization algorithms are, unfortunately, difficult to understand and communicate. We provide a dynamic programming (DP) formulation based on the idea of optimally allocating a given echelon-inventory level between the upstream stage and the downstream series system. This formulation yields an algorithm that can be improved by incorporating gradient updates. We develop a simple, near-optimal, heuristic that follows from the DP formulation by judiciously selecting a common holding cost for the downstream stages. The heuristic calls for solving a single newsvendor problem per stage and is very accessible to students and practitioners. The need to develop accurate and accessible spreadsheet-based heuristics was recently identified by Shang and Song [13] who develop a heuristic based on solving two newsvendor problems per stage. We evaluate our heuristic and compare it to that of Shang and Song by testing it on the set of test problems in Gallego and Zipkin [8] and Shang and Song [13] and in additional experiments designed to test the performance when different stages have different lead times. Our numerical results indicate that our heuristic is near optimal with an average error that is lower than the Shang and Song heuristic. Finally, we provide an approximate distribution-free bound that accurately reflects the sensitivity of the optimal average cost to changes in system parameters.

Consider a series system consisting of $J$ stages as illustrated in the figure. Stage $j<J$ procures from Stage $j+1$ and Stage $J$ replenishes from an outside supplier with ample stock. Customer demand occurs only at Stage 1 and follows a (compound) Poisson process, $\{D(t), t \geq 0\}$ with arrival rate $\lambda$ and random demand size $X$ with $E\left[X^{2}\right]<\infty$. It takes $L_{j}$ units of time for a unit to arrive at Stage $j$ once it is released by its predecessor.

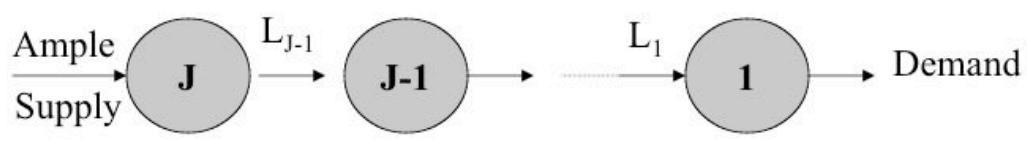

Unsatisfied demand is backordered at each stage but only Stage 1 incurs a linear backorder penalty cost $p$, per unit, per unit time. We assume without loss of generality that each stage adds value as the item moves through the supply chain, so echelon holding costs $h_{j}^{e}$ are positive. The local holding cost for stage $j$ is $h_{j}=h^{e}[j, J] \equiv \sum_{i=j}^{J} h_{i}^{e}$, where sums over empty sets will be defined as zero. In particular, $h_{J+1}=0$. The system is operated under continuous review, so an order is placed every time a demand occurs. As pointed out by Zipkin [14], this is justified for expensive and/or slow moving items.

The following random variables describe the state of Stage $j$ in equilibrium:

$$
\begin{aligned}
D_{j} & =\text { leadtime demand } \\
I_{j} & =\text { on-hand inventory } \\
B_{j} & =\text { backorders. }
\end{aligned}
$$


The total long run average cost for any policy can be expressed as

$$
E\left[\sum_{k=1}^{J} h_{k} I_{k}+p B_{1}+\sum_{k=2}^{J} h_{k} D_{k-1}\right]
$$

Optimality of an echelon base stock policy $\left(s_{J}, \ldots, s_{1}\right)$ for this series system is well known (see Federgruen and Zipkin [4], Chen and Zheng [2] and Zipkin [14], and the original work by Clark and Scarf [3]). Under this policy, the manager continuously monitors the echelon inventory order position at each stage and places an order from Stage $j+1$ to bring it up to $s_{j}$ whenever it is below this level. An echelon base stock policy $\left(\tilde{s}_{J}, \tilde{s}_{J-1}, \ldots, \tilde{s}_{1}\right)$ is equivalent to $\left(s_{J}, \ldots, s_{1}\right)$ where $s_{j}=\min \left(\tilde{s}_{J}, \ldots, \tilde{s}_{j}\right)$, so there exists an echelon base-stock policy satisfying the property $s_{J} \geq s_{J-1} \geq \ldots \geq s_{1}$. Let $s_{o} \equiv 0$ and let $s_{j}^{\prime} \equiv s_{j}-s_{j-1}, j \geq 1$ denote the local base stock level at stages $j=1, \ldots, J$. This local base stock policy is equivalent to the echelon base stock policy (see Axsater [1] Propositions 1 and 2 or Zipkin [14] pg 306). The following recursion gives the steady state distribution of the local on-hand inventory $I_{k}$ and the backorder $B_{k}$ at Stage $k$ starting with $B_{J+1}=0$.

$$
I_{k}=\left(s_{k}^{\prime}-D_{k}-B_{k+1}\right)^{+} \quad B_{k}=\left(B_{k+1}+D_{k}-s_{k}^{\prime}\right)^{+} \quad k=J, \ldots, 1 .
$$

Then, the total long-run average cost can be expressed as

$$
g_{J}\left(s_{J}, \ldots, s_{1}\right)=E\left[\sum_{k=1}^{J} h_{k}\left(s_{k}^{\prime}-D_{k}-B_{k+1}\right)^{+}+p B_{1}+\sum_{k=2}^{J} h_{k} D_{k-1}\right] .
$$

Consider the sub-system consisting of Stages $\{j, \ldots, 1\}$ for some $j \in\{1, \ldots, J-1\}$ and assume that Stage $j$ replenishes its inventory from an external supplier with ample supply. This subsystem is equivalent to the original $J$ stages series system with $D_{k} \equiv 0, h_{k} \equiv 0$ for all $k>j$. Let $g_{j}\left(s_{j}, \ldots, s_{1}\right)$ be the long-run total average cost for an echelon base-stock policy $\left(s_{j}, \ldots, s_{1}\right)$. Then $g_{j}\left(s_{j}, \ldots, s_{1}\right)$ can be obtained via equations $(2)$ and (3) by replacing $J$ by $j$ starting the computations with $B_{j+1}=0$.

The next result provides a link between the cost of the different sub-systems.

\section{Proposition 1}

$$
g_{1}(s)=E\left[h_{1}\left(s-D_{1}\right)^{+}+p\left(D_{1}-s\right)^{+}\right]
$$

and for $j=2, \ldots, J$

$$
g_{j}\left(s_{j}, \ldots, s_{1}\right)=E\left[h_{j}\left(s_{j}^{\prime}-D_{j}\right)^{+}+g_{j-1}\left(\min \left(s_{j-1}, s_{j}-D_{j}\right), s_{j-2}, \ldots, s_{1}\right)+h_{j} D_{j-1}\right] .
$$

Proof. Let $g_{o}(s)=p(-s)^{+}$, then for $j=1$,

$$
\begin{aligned}
g_{1}(s) & =E\left[h_{1}\left(s-D_{1}\right)^{+}+p B_{1}\right] \\
& =E\left[h_{1}\left(s-D_{1}\right)^{+}+p\left(D_{1}-s\right)^{+}\right] \\
& =E\left[h_{1}(s-D) 1\right)^{+}+g_{o}\left(\min \left(0, s_{1}-D_{1}\right)\right) .
\end{aligned}
$$


This shows that the result holds for $j=1$. Suppose the result holds for some $j<J$. We now show that it holds for $j+1$. Clearly from Equation (3),

$g_{j+1}\left(s_{j+1}, s_{j}, \ldots, s_{1}\right)=E h_{j+1}\left(s_{j+1}^{\prime}-D_{j+1}\right)^{+}+E\left[\sum_{k=1}^{j} h_{k}\left(s_{k}^{\prime}-D_{k}-B_{k+1}\right)^{+}+p B_{1}+\sum_{k=2}^{j} h_{k} D_{k-1}\right]+h_{j+1} E D_{j}$.

The term in square brackets looks exactly as the definition of $g_{j}\left(s_{j}, \ldots, s_{1}\right)$ except that $B_{j+1}=$ $\left(D_{j+1}-s_{j+1}^{\prime}\right)^{+}$instead of 0 . Thus, the echelon base-stock level at Stage $j$ is given by $s_{j}-B_{j+1}=$ $\min \left(s_{j}, s_{j+1}-D_{j+1}\right)$ instead of $s_{j}$ and this justifies (4) for $j+1$, completing the proof.

We now provide a recursion to find the optimal expected cost at each stage for an arbitrary echelon base stock level, as well as an algorithm to find optimal base-stock policies for each subsystem $\{j, \ldots, 1\}$ for $j=1, \ldots, J$.

Let $c_{1}(s)=g_{1}(s)$ and for $j=2, \ldots, J$ define

$$
c_{j}(s)=\min _{x \in\{0, \ldots, s\}} c_{j}(x ; s)
$$

recursively, via

$$
c_{j}(x ; s)=E\left[h_{j}\left(x-D_{j}\right)^{+}+c_{j-1}\left(\min \left(s-x, s-D_{j}\right)\right)+h_{j} D_{j-1}\right] .
$$

Let $\mathcal{N}=\{0,1, \ldots$,$\} be the set of non-negative integers and let$

$$
s_{j}^{*} \equiv \min \left\{s \in \mathcal{N}: \Delta c_{j}(s)>h_{j+1}\right\} \quad \text { for } \quad j=1, \ldots, J
$$

We will show that $c_{j}(s)$ is the long run average cost of optimally managing the sub-system $\{j, \ldots, 1\}$ given echelon base stock level $s$ and that optimal base stock levels are given by (7). Before we prove this result formally, we will provide an intuitive link between equations (6) and (5). Suppose that we have computed $c_{j}(\cdot)$ and consider the sub-system $\{j+1, \ldots, 1\}$. Our goal is to compute $c_{j+1}(\cdot)$ from the knowledge of $c_{j}(\cdot)$. To link the two sub-systems, we decompose the echelon base stock level $s$ of sub-system $\{j+1, \ldots, 1\}$ by allocating $x$ units to Stage $j+1$ and $s-x$ units for sub-system $\{j, \ldots, 1\}$. Given this allocation, the net inventory at Stage $j+1$ will be $\left(x-D_{j+1}\right)^{+}$ which accrues at cost rate $h_{j+1}$. Since Stage $j+1$ will face a shortage when $D_{j+1}-x>0$, the effective echelon inventory for sub-system $\{j, \ldots, 1\}$ is $s-x-\left(D_{j+1}-x\right)^{+}=\min \left(s-x, s-D_{j+1}\right)$. Thus, a finite local base stock level at Stage $j+1$ imposes an externality to the sub-system $\{j, \ldots, 1\}$ whose expected cost is now $E c_{j}\left(\min \left(s-x, s-D_{j+1}\right)\right)$. As a result, when we allocate $x \leq s$ units of local base stock level to Stage $j+1$, the cost of managing a series system with $j+1$ stages is given by $(6)$.

Theorem 1 1) $c_{j}(s)$ is convex in $s$ for all $j=1, \ldots, J$ and given by (5) for $j=2, \ldots, J$; 2) $x_{j}(s)=\left(s-s_{j-1}^{*}\right)^{+}$minimizes $c_{j}(x ; s)$ for $\left.j=2, \ldots, J ; 3\right)$ The echelon base stock policy $\left(s_{j}^{*}, \ldots, s_{1}^{*}\right)$ is optimal and $c_{j}\left(s_{j}^{*}\right)$ is the optimal expected cost for sub-system $\{j, \ldots, 1\}, j=1, \ldots, J$. 
Proof. For $j=1$, the optimal cost of managing the subsystem given echelon base stock level $s$ is simply $c_{1}(s)=g_{1}(s)$ since there is nothing to optimize. Notice that $\Delta c_{1}(s) \equiv c_{1}(s+1)-c_{1}(s)=$ $\left(h_{1}+p\right) \operatorname{Pr}\left(D_{1} \leq s\right)-p$ is non-decreasing in $s$ so $c_{1}(s)$ is convex. The largest optimal base stock level is given by $s_{1}^{*}=\min \left\{s \in \mathcal{N}: \Delta c_{1}(s)>0\right\}$ which is consistent with (7) since $h_{2}=0$ for the sub-system consisting only of Stage 1 . As a result $c_{1}\left(s_{1}^{*}\right)$ is the optimal expected cost for the sub-system consisting of Stage 1 only. This establishes Parts 1 and 3 for $j=1$.

Consider now the sub-system $\{2,1\}$ and notice that

$$
\begin{aligned}
c_{2}(s) & =\min _{s_{1} \in\{0, \ldots, s\}} g_{2}\left(s, s_{1}\right) \\
& =\min _{s_{1} \in\{0, \ldots, s\}} E\left[h_{2}\left(s-s_{1}-D_{2}\right)^{+}+g_{1}\left(\min \left(s_{1}, s-D_{2}\right)\right)+h_{2} D_{1}\right] \\
& =\min _{x \in\{0, \ldots, s\}} E\left[h_{2}\left(x-D_{2}\right)^{+}+c_{1}\left(\min \left(s-x, s-D_{2}\right)\right)+h_{2} D_{1}\right] \\
& =\min _{x \in\{0, \ldots, s\}} c_{2}(x ; s),
\end{aligned}
$$

where the second equality is from Equation (4) and the third is by substituting $x$ for $s-s_{1}$. Therefore, $c_{2}(s)$ is given by $(5)$. Now let $\Delta c_{2}(x ; s) \equiv c_{2}(x+1 ; s)-c_{2}(x ; s)$. Then,

$$
\Delta c_{2}(x ; s)=\left[h_{2}-\Delta c_{1}(s-x-1)\right] \operatorname{Pr}\left(D_{2} \leq x\right) \text {. }
$$

Notice that $\Delta c_{2}(x ; s)=0$ for all $x<0$ on account of $D_{2} \geq 0$. The convexity of $c_{1}$ implies that $\Delta c_{1}(s-x-1)$ is decreasing in $x$. As a consequence, $\Delta c_{2}(x ; s)$ has at most one sign change over the range $x \in\{0, \ldots, s\}$ and this would have to be from - to + . From Equation (7), $s_{1}^{*}$ is the smallest non-negative integer $y$ such that $\Delta c_{1}(y)>h_{2}$. Notice that $s_{1}^{*}$ is the largest minimizer of the newsvendor problem with holding cost $h_{1}-h_{2}$, backorder cost $p+h_{2}$ and demand $D_{1}$. In particular, $s_{1}^{*}$ is independent of the distribution of $D_{2}$. We have shown that $x=\left(s-s_{1}^{*}\right)^{+}$is a minimizer of $c_{2}(\cdot ; s)$ establishing Part 2 for $j=2$. This result implies that allocating $s_{1}^{*}$ units of echelon base stock level to Stage 1 is optimal when $s \geq s_{1}^{*}$. Therefore, we have

$$
\begin{aligned}
c_{2}(s) & =c_{2}\left(\left(s-s_{1}^{*}\right)^{+} ; s\right)=E\left[h_{2}\left(\left(s-s_{1}^{*}\right)^{+}-D_{2}\right)^{+}+c_{1}\left(\min \left(s_{1}^{*}, s-D_{2}\right)\right)+h_{2} D_{1}\right] \\
& =E\left[h_{2}\left(s-s_{1}^{*}-D_{2}\right)^{+}+c_{1}\left(\min \left(s_{1}^{*}, s-D_{2}\right)\right)+h_{2} D_{1}\right]
\end{aligned}
$$

where the last equation follows since $\left(x^{+}-a\right)^{+}=(x-a)^{+}$when $a \geq 0$. To see that $c_{2}$ is convex, notice that

$$
\Delta c_{2}(s)=h_{2} \operatorname{Pr}\left(D_{2} \leq s-s_{1}^{*}\right)+\sum_{x=\left(s+1-s_{1}^{*}\right)^{+}}^{\infty} \Delta c_{1}(s-x) \operatorname{Pr}\left(D_{2}=x\right)
$$

Let $\Delta^{2} c_{2}(s) \equiv \Delta c_{2}(s+1)-\Delta c_{2}(s)$, then

$$
\Delta^{2} c_{2}(s)=\sum_{x=\left(s+2-s_{1}^{*}\right)^{+}}^{\infty} \Delta^{2} c_{1}(s-x) \operatorname{Pr}\left(D_{2}=x\right)+\left[h_{2}-\Delta c_{1}\left(s_{1}^{*}-1\right)\right] \operatorname{Pr}\left(D_{2}=s+1-s_{1}^{*}\right) .
$$

Now, $\Delta^{2} c_{1}(s-x) \geq 0$ on account of the convexity of $c_{1}$, so the first term is non-negative. The second term is also non-negative by the definition of $s_{1}^{*}$. This establishes Part 1 for $j=2$. Recall 
that $h_{3} \equiv 0$ for sub-system $\{2,1\}$. Hence, the minimizer $s_{2}^{*}$ of $c_{2}(s)$ is given by Equation $(7)$ with $h_{3}=0$. With this final observation, we have shown that $\left(s_{2}^{*}, s_{1}^{*}\right)$ is an optimal echelon base-stock policy for sub-system $\{2,1\}$ with optimal expected cost $c_{2}\left(s_{2}^{*}\right)$ establishing Part 3 for $j=2$.

Assume now that all three statements are true for sub-system $\{j, \ldots, 1\}$ for some $j<J$. We now add Stage $j+1$ with local holding cost $h_{j+1}$. Then,

$$
\begin{aligned}
c_{j+1}(s) & =\min _{0 \leq s_{1} \leq \ldots \leq s_{j} \in\{0, \ldots, s\}} g_{j+1}\left(s, s_{j}, \ldots, s_{1}\right) \\
& =\min _{0 \leq s_{1} \leq \ldots \leq s_{j} \in\{0, \ldots, s\}} E\left[h_{j+1}\left(s-s_{j}-D_{j+1}\right)^{+}+g_{j}\left(\min \left(s_{j}, s-D_{j+1}\right), s_{j-1}, \ldots, s_{1}\right)+h_{j+1} D_{j}\right] \\
& =\min _{x \in\{0, \ldots, s\}} E\left[h_{j+1}\left(x-D_{j+1}\right)^{+}+\min _{s_{1}, \ldots, s_{j-1}} g_{j}\left(\min \left(s-x, s-D_{j+1}\right), s_{j-1}, \ldots, s_{1}\right)+h_{j+1} D_{j}\right] \\
& =\min _{x \in\{0, \ldots, s\}} E\left[h_{j+1}\left(x-D_{j+1}\right)^{+}+c_{j}\left(\min \left(s-x, s-D_{j+1}\right)\right)+h_{j+1} D_{j}\right] \\
& =\min _{x \in\{0, \ldots, s\}} c_{j+1}(x ; s),
\end{aligned}
$$

so $c_{j+1}(s)$ is given by $(5)$. Notice that $c_{j+1}(x+1 ; s)-c_{j+1}(x, s)$ is non-zero only when $D_{j+1} \leq x$ and is equal, in this case, to $h_{j+1}-c_{j}(s-x-1)+c_{j}(s-x)=h_{j+1}-\Delta c_{j}(s-x-1)$. Consequently,

$$
\Delta c_{j+1}(x ; s)=\left[h_{j+1}-\Delta c_{j}(s-x-1)\right] \operatorname{Pr}\left(D_{j+1} \leq x\right) .
$$

Now, since $c_{j}$ is convex it follows that $\Delta c_{j+1}(x ; s)$ has at most one sign change and this must be from - to + . Since the sign change occurs at $\left(s-s_{j}^{*}\right)$ when $s \geq s_{j}^{*}$, it follows that $x_{j+1}(s)=\left(s-s_{j}^{*}\right)^{+}$ minimizes $c_{j+1}(x ; s)$ so $c_{j+1}(s)=c_{j+1}\left(\left(s-s_{j}^{*}\right)^{+} ; s\right)$ establishing Part 2 for $j+1$. This result implies that allocating $s_{j}^{*}$ units of echelon base stock level to stage $j$ is optimal when $s \geq s_{j}^{*}$. Therefore, we have

$$
c_{j+1}(s)=E\left[h_{j+1}\left(s-s_{j}^{*}-D_{j+1}\right)^{+}+c_{j}\left(\min \left(s_{j}^{*}, s-D_{j+1}\right)\right)+h_{j+1} D_{j}\right] .
$$

The convexity of $c_{j+1}$ now follows the exact argument used to establish the convexity of $c_{2}$. Indeed,

$\Delta^{2} c_{j+1}(s)=\sum_{x=\left(s+2-s_{j}^{*}\right)^{+}}^{\infty} \Delta^{2} c_{j}(s-x) \operatorname{Pr}\left(D_{j+1}=x\right)+\left[h_{j+1}-\Delta c_{j}\left(s_{j}^{*}-1\right)\right] \operatorname{Pr}\left(D_{j+1}=s+1-s_{j}^{*}\right) \geq 0$

because $\Delta^{2} c_{j} \geq 0$ and by the definition of $s_{j}^{*}$. This proves Part 1 for $j+1$. Recall that $h_{j+2}=0$ for sub-system $\{j+1, \ldots, 1\}$, so it follows that the minimizer $s_{j+1}^{*}$ of $c_{j+1}(s)$ is given by Equation (7) with $h_{j+2}=0$, implying that $\left(s_{j+1}^{*}, \ldots, s_{1}^{*}\right)$ is an optimal echelon base stock policy for sub-system $\{j+1, \ldots, 1\}$ and that $c_{j+1}\left(s_{j+1}^{*}\right)$ is the optimal expected cost for this sub-system. This establishes Part 3 for $j+1$ and concludes the induction argument for $j+1$ and hence the proof.

Remark: Notice that the definition of $s_{j}^{*}$ changes as we go from sub-system $\{j, \ldots, 1\}$ to sub-system $\{j+1, \ldots, 1\}$ because $h_{j+1}=0$ for sub-system $\{j, \ldots, 1\}$ but $h_{j+1}>0$ for sub-system $\{j+1, \ldots, 1\}$. However, the echelon base stock policy $\left(s_{j}^{*}, \ldots, s_{1}^{*}\right)$ does not change after we add Stage $j+1$ and in the course of the algorithm we need to find each $s_{j}^{*}$ only once. Finally, notice that $c_{J+1}\left(s_{J}^{*}\right)=c_{J}\left(s_{J}^{*}\right)$ on account of $h_{J+1}=0$ and $D_{J+1} \equiv 0$. 
Optimal echelon base stock levels can also be found through solving the traditional recursive optimization for $j=1,2, \ldots, J$. This formulation is based on echelon cost accounting.

$$
\begin{aligned}
C_{j}(y) & =E\left\{h_{j}^{e}\left(y-D_{j}\right)+C_{j-1}\left(\min \left[y-D_{j}, s_{j-1}^{*}\right]\right)\right\} \\
s_{j}^{*} & \equiv \max \left\{y: C_{j}(y) \leq C_{j}(x) \text { for all } x \neq y\right\}
\end{aligned}
$$

where $C_{0}(y)=\left(p+h_{1}\right)[y]^{-}$, see Chen and Zheng [2] and Gallego and Zipkin [8]. The optimal system wide average cost is given by $C_{J}\left(s_{J}^{*}\right)$. We now verify that the new algorithm produces the same echelon base stock levels as the traditional algorithm and find an explicit relationship between the $C_{j}$ s and the $c_{j}$ s.

Proposition 2 1) $C_{j}(s)=c_{j}(s)-h_{j+1} E\left(s-D_{j}\right)$; 2) $C_{J}(s)=c_{J}(s)$; 3) $\Delta C_{j}(s)=\Delta c_{j}(s)-h_{j+1}, s o$ $s_{j}^{*}$ minimizes $C_{j}(s)$.

Proof. The proof is based on an induction argument. For the case $j=1$, we have $C_{1}(s)=$ $E\left[h_{1}^{e}\left(s-D_{1}\right)+\left(p+h_{1}\right)\left(D_{1}-s\right)^{+}\right]=E\left[h_{1}^{e}\left(s-D_{1}\right)-h_{1}\left(s-D_{1}\right)+h_{1}\left(s-D_{1}\right)+\left(p+h_{1}\right)\left(D_{1}-s\right)^{+}\right]=$ $c_{1}(s)+E\left[h_{1}^{e}\left(s-D_{1}\right)-h_{1}\left(s-D_{1}\right)\right]=c_{1}(s)-h_{2} E\left(s-D_{1}\right)$. Suppose the result holds for $j$, then $C_{j+1}(s)=E\left[h_{j+1}^{e}\left(s-D_{j+1}\right)+C_{j}\left(\min \left(s_{j}^{*}, s-D_{j+1}\right)\right)=E\left[h_{j+1}^{e}\left(s-D_{j+1}\right)+c_{j}\left(\min \left(s_{j}^{*}, s-D_{j+1}\right)\right)-\right.\right.$ $\left.h_{j+1} E\left(\min \left(s_{j}^{*}, s-D_{j+1}\right)-D_{j}\right)\right] \pm h_{j+1} E\left(s-s_{j}^{*}-D_{j+1}\right)^{+}=c_{j+1}(s)-h_{j+2} E\left(s-D_{j+1}\right)$. Part 2 follows directly from the fact that $h_{J+1} \equiv 0$. Part 3 follows directly from Part 1 and the definition of $s_{j}^{*}$.

Remark: Our result also allows us to interpret $C_{j}(s)$ as $c_{j}(s)$ plus the inventory in transit to Stage $j$ minus an echelon inventory credit at rate $h_{j+1}$. In our opinion, the interpretation of $c_{j}(s)$ is easier to understand.

\section{Algorithm with Gradient Updates}

An algorithm strictly based on Equations (5), (6) and (7) requires computing $c_{j}(x ; s)$ for each $x \in\{0, \ldots, s\}$ and a minimization over this set to compute $c_{j}(s)$. Evaluating $c_{j}(x ; s)$ for each $x$ takes computational work proportional to $\max (s-x, x)$. Therefore, evaluating $c_{j}(x ; s)$ for all $x \in\{0, \ldots, s\}$ requires work proportional to $s^{2}$. The minimizing over $x \in\{0, \ldots, s\}$ does not add to the complexity because the order of complexity for searching the minimizer is $s \ln (s)$. In summary, computational work to evaluate $c_{j}(s)$ is proportional to $s^{2}$. Computing $c_{j}(0), \ldots, c_{j}\left(s_{j}^{*}\right)$ takes work proportional to $\left(s_{j}^{*}\right)^{3}$. Adding this work over all the stages gives us the computational complexity of the algorithm. The complexity for the traditional algorithm without the gradient updates is similar.

The next proposition establishes the link between $\Delta c_{j+1}(x)$ and $\Delta c_{j}(x)$ that requires considerably less work. Indeed, the work required to compute $\Delta c_{j}(s)$, via Equation $(10)$, is proportional to $s$, so the work required to compute $s_{j}^{*}$ is proportional to $\left(s_{j}^{*}\right)^{2}$. Adding the work over all the stages gives us the complexity of the algorithm with gradient updates. 
Proposition 3 For $j=1, \ldots, J$, we have

$$
\Delta c_{j+1}(s)=h_{j+1} \operatorname{Pr}\left(D_{j+1} \leq\left(s-s_{j}^{*}\right)^{+}\right)+\sum_{k=0}^{\min \left(s, s_{j}^{*}-1\right)} \Delta c_{j}(k) \operatorname{Pr}\left(D_{j+1}=s-k\right)-p \operatorname{Pr}\left(D_{j+1}>s\right) .
$$

Proof. Since $c_{j+1}(s)=E\left[h_{j+1}\left(\left(s-s_{j}^{*}\right)^{+}-D_{j+1}\right)^{+}+h_{j+1} E D_{j}+c_{j}\left(\min \left(s_{j}^{*}, s-D_{j+1}\right)\right)\right]$, after some algebra we obtain:

$$
\Delta c_{j+1}(s)=h_{j+1} \operatorname{Pr}\left(D_{j+1} \leq s-s_{j}^{*}\right)+\sum_{k=\left(s+1-s_{j}^{*}\right)^{+}}^{\infty} \Delta c_{j}(s-k) \operatorname{Pr}\left(D_{j+1}=k\right) .
$$

If $s<s_{j}^{*}$, the first term is zero and

$$
\begin{aligned}
\Delta c_{j+1}(s) & =E \Delta c_{j}\left(s-D_{j+1}\right)=\sum_{k=0}^{\infty} \Delta c_{j}(s-k) \operatorname{Pr}\left(D_{j+1}=k\right) \\
& =\sum_{k=0}^{s} \Delta c_{j}(s-k) \operatorname{Pr}\left(D_{j+1}=k\right)-\operatorname{pPr}\left(D_{j+1}>s\right) \\
& =\sum_{k=0}^{s} \Delta c_{j}(k) \operatorname{Pr}\left(D_{j+1}=s-k\right)-p \operatorname{Pr}\left(D_{j+1}>s\right),
\end{aligned}
$$

where the last two equations follow from $\Delta c_{j}(s)=-p$ for $s<0$. The last equation is equivalent to (10) for $s<s_{j}^{*}$. Next we show the result for $s \geq s_{j}^{*}$. In this case,

$$
\Delta c_{j+1}(s)=h_{j+1} \operatorname{Pr}\left(D_{j+1} \leq s-s_{j}^{*}\right)+\sum_{k=s+1-s_{j}^{*}}^{\infty} \Delta c_{j}(s-k) \operatorname{Pr}\left(D_{j+1}=k\right) .
$$

By noticing that $\Delta c_{j}(s)=-p$ for $s<0$, we can rewrite the difference as

$$
\Delta c_{j+1}(s)=h_{j+1} \operatorname{Pr}\left(D_{j+1} \leq s-s_{j}^{*}\right)+\sum_{k=0}^{s_{j}^{*}-1} \Delta c_{j}(k) \operatorname{Pr}\left(D_{j+1}=s-k\right)-p \operatorname{Pr}\left(D_{j+1}>s\right) .
$$

This is equivalent to Equation (10) for $s \geq s_{j}^{*}$, concluding the proof.

Next we describe an algorithm to obtain an optimal echelon base stock policy and the optimal expected cost.

$s_{1}^{*} \leftarrow \min \left\{y \in \mathcal{N}: \Delta c_{1}(y)>h_{2}\right\}$,

$c_{J}(0) \leftarrow \sum_{i=1}^{J} h_{i+1} E D_{i}+p E D[1, J]$

FOR $j=1$ to $J-1 \mathrm{DO}$

$$
\begin{aligned}
& \Delta c_{j+1}(s) \leftarrow h_{j+1} \operatorname{Pr}\left(D_{j+1} \leq\left(s-s_{j}^{*}\right)^{+}\right)+\sum_{k=0}^{\min \left(s, s_{j}^{*}-1\right)} \Delta c_{j}(k) \operatorname{Pr}\left(D_{j+1}=s-k\right)-p \operatorname{Pr}\left(D_{j+1}>s\right) . \\
& s_{j+1}^{*} \leftarrow \min \left\{y \in \mathcal{N}: \Delta c_{j+1}(y)>h_{j+2}\right\} .
\end{aligned}
$$

END

$\operatorname{PRINT}\left(s_{J}^{*}, \ldots, s_{1}^{*}\right)$ and $c_{J}\left(s_{J}^{*}\right)=c_{J}(0)+\sum_{y=0}^{s_{J}^{*}-1} \Delta c_{J}(y)$. 


\section{Newsvendor Bounds and Heuristics}

The DP formulation enables the design of a fast algorithm based on gradient updates. Yet, both the new and the traditional formulation are difficult to explain to non-mathematically oriented students and practitioners and do not allow for sensitivity analysis. We now provide a heuristic that can be implemented in a spreadsheet by solving one newsvendor problem per stage.

Consider the sub-system $\{j+1, \ldots, 1\}$, for some $j \in\{1, \ldots, J-1\}$ at the time we are allocating $s$ between Stage $j+1$ and sub-system $\{j, \ldots, 1\}$. What complicates the problem of minimizing $c_{j+1}(x ; s)$ is the fact that the holding costs $h_{k}, k=j+1, \ldots, 1$ are increasing. To see how this simplifies when the downstream costs are equal consider what happens when $h_{j+1}<H_{j}=h_{j}=$ $h_{j-1}=\ldots=h_{1}$. We will use the notation $c_{j+1}\left(x ; s \mid H_{j}\right)$ to denote the cost of allocating $x$ units to Stage $j+1$ and $s-x$ units to sub-system $\{j, \ldots, 1\}$ where the downstream holding costs are $H_{j}$. When it costs the same to hold stock at stages $j, \ldots, 1$ it is optimal to hold stock only at Stage 1 since this affords better protection against backorders. This observation implies that we can collapse stages $\{j, \ldots, 1\}$ into a single stage with demand $D[1, j]$ and holding cost $H_{j}$. This reduces the problem to a two stage problem. Indeed,

$$
c_{j+1}\left(x ; s \mid H_{j}\right)=h_{j+1}\left(x-D_{j+1}\right)^{+}+h_{j+1} E D_{j}+H_{j} \sum_{k=1}^{j-1} E D_{k}+E G_{j}\left(\min \left(s-x, s-D_{j+1}\right) \mid H_{j}\right),
$$

where $G_{j}\left(s \mid H_{j}\right)=H_{j} E(s-D[1, j])^{+}+p E(D[1, j]-s)^{+}$. Consequently, the first difference is given by

$$
\Delta c_{j+1}\left(x ; s \mid H_{j}\right)=\left[h_{j+1}-\Delta G_{j}\left(s-x-1 \mid H_{j}\right)\right] \operatorname{Pr}\left(D_{j+1} \leq x\right) .
$$

Notice also that $\Delta G_{j}\left(s \mid H_{j}\right) \equiv G_{j}\left(s+1 \mid H_{j}\right)-G_{j}\left(s \mid H_{j}\right)=\left(p+H_{j}\right) \operatorname{Pr}(D[1, j] \leq s)-p$ and consequently,

$$
s_{j}^{*}\left(H_{j}\right) \equiv \min \left\{s \in \mathcal{N}:\left(p+H_{j}\right) \operatorname{Pr}(D[1, j] \leq s)>p+h_{j+1}\right\} .
$$

In other words, $s_{j}^{*}\left(H_{j}\right)$ is the largest minimizer of a newsvendor problem with demand $D[1, j]$, holding cost $H_{j}-h_{j+1}$, backorder penalty cost $p+h_{j+1}$ and that the solution is independent of the demand $D_{j+1}$. Substituting $x=\left(s-s_{j}^{*}\left(H_{j}\right)\right)^{+}$, we obtain:

$$
\begin{aligned}
c_{j+1}\left(s \mid H_{j}\right) & =h_{j+1}\left(s-s_{j}^{*}\left(H_{j}\right)-D_{j+1}\right)^{+}+h_{j+1} E D_{j}+H_{j} \sum_{k=1}^{j-1} E D_{k} \\
& +E G_{j}\left(\min \left(s_{j}^{*}(H), s-D_{j+1} \mid H_{j}\right) .\right.
\end{aligned}
$$

Notice that the heuristic is intimately related to the dynamic programming formulation; it follows the same steps, but that of finding $s_{j}^{*}$ is greatly simplified by the assumption of equal holding costs for the downstream sub-system. We can improve on the cost estimate in Equation (14) by using the actual pipeline cost $\sum_{k=1}^{j} h_{k+1} E D_{k}$ instead of the approximation $h_{j+1} E D_{j}+H_{j} \sum_{k=1}^{j-1} E D_{k}$.

Consider now the general case where $h_{j}<h_{j-1}<\ldots<h_{1}$. Shang and Song (2003) increase the holding costs of stages $j, \ldots, 2$ to $H_{j}=h_{1}$. For this sub-system, the cost of allocating $x$ units to 
$j+1$ and $s$ units to $j$ is given by $c_{j+1}\left(x ; s \mid h_{1}\right)$ as defined in (11) and the optimal echelon base-stock level for Stage $j$ is given by $s_{j}^{*}\left(h_{1}\right)$. Similarly, they decrease the holding cost of stages $j-1, \ldots, 1$ to $H_{j}=h_{j}$. The resulting sub-system has expected cost $c_{j+1}\left(x ; s \mid h_{j}\right)$ and optimal echelon base-stock level $s_{j}^{*}\left(h_{j}\right)$. Shang and Song thus solve two newsvendor problems per stage to obtain $s_{j}^{*}\left(h_{1}\right)$ and $s_{j}^{*}\left(h_{j}\right), j=1, \ldots, J$ and show that $s_{j}^{*}\left(h_{1}\right) \leq s_{j}^{*} \leq s_{j}^{*}\left(h_{j}\right)$. They develop a heuristic for the original system by either truncating or rounding the average $\left(s_{j}^{*}\left(h_{1}\right)+s_{j}^{*}\left(h_{j}\right)\right) / 2$.

We propose a new heuristic that consists of solving a single newsvendor problem per stage based on the approximate holding cost rate

$$
H_{j}=h_{j}^{G O} \equiv \sum_{k=1}^{j} \frac{L_{k}}{L[1, j]} h_{k} .
$$

The idea is based on adding the holding cost as the product goes through the stages without delay and then dividing by the total lead time that it spends before reaching the end customer. In this way, we obtain an approximate holding cost rate for each stage and solve the system $c_{j+1}\left(x ; s \mid h_{j}^{G 0}\right)$ obtaining $s_{j}^{*}\left(h_{j}^{G 0}\right)$ for $j=1, \ldots, J$. We explored other weighting strategies as discussed in the last paragraph of the numerical study.

Proposition 4 For any given $j$ and $s$ we have:

1. $c_{j}\left(s \mid h_{j}\right) \leq c_{j}\left(s \mid h_{j}^{G O}\right) \leq c_{j}\left(s \mid h_{1}\right)$,

2. $s_{j}^{*}\left(h_{1}\right) \leq s_{j}^{*}\left(h_{j}^{G O}\right) \leq s_{j}^{*}\left(h_{j}\right)$,

3. $G_{j}\left(s \mid h_{j}^{G O}\right) \leq \sqrt{p h_{j}^{G O}} \sqrt{\lambda L[1, j] E\left[X^{2}\right]}$.

Proof. Notice that $h_{j} \leq h_{j}^{G O} \leq h_{1}$. Part 1 follows immediately from this inequality. We also have $\Delta G_{j}\left(s \mid h_{j}\right) \geq \Delta G_{j}\left(s \mid h_{j}^{G O}\right) \geq \Delta G_{j}\left(s \mid h_{1}\right)$ since $h_{j} \leq h_{j}^{G O} \leq h_{1}$. This implies Part 2. Finally Part 3 is the distribution-free bound in Gallego and Moon [5] and Scarf [11].

The last proposition imply that if the bounds in Part 1 of Proposition 4 are tight then $s_{j}^{*}\left(h_{j}^{G O}\right)$ would be a close to optimal heuristic. We will illustrate the accuracy of this heuristic in the following section. If our heuristic is close-to-optimal, the cost of managing the series system can also be bounded by the distribution-free upper bound

$$
c_{J}\left(s_{J}^{*}\right)=c_{J+1}\left(s_{J}^{*}\right) \simeq c_{J+1}\left(s_{J}^{*}\left(h_{J}^{G 0}\right) \mid h_{J}^{G 0}\right) \leq \sqrt{p\left(h_{1} L_{1}+\ldots+h_{J} L_{J}\right) \lambda E X^{2}}+\sum_{i=1}^{J-1} h_{i+1} E D_{i} .
$$

This formula contains all of the parameters of the problem. A simple sensitivity analysis reveals that (1) the system cost is proportional to $\sqrt{p},(2)$ downstream leadtimes have a larger impact on system performance than upstream leadtimes, (3) upstream echelon holding cost rates have a larger impact on the system performance than downstream echelon holding cost rates, (4) the system cost is proportional to $\sqrt{\lambda}$ and proportional to $\sqrt{E\left[X^{2}\right]}$, recall that $X$ is the random demand size. Note that it is possible to approximate the optimal expected cost for each stage by using the normal 
approximation. This approximation would result in a formula of the form $c_{j}\left(s_{j}^{*}\right) \simeq\left(p+H_{j}\right) \sigma \phi(z)$, where $z=\Phi^{-1}\left(\left(p+h_{j+1}\right) /\left(p+H_{j}\right)\right)$ for $j=1, \ldots, J$ and $\sigma$ is the standard deviation of $D[1, j]$.

This type of parametric analysis enables a near characterization of system performance. Some system design issues may require investments in new processing plans or quicker but more expensive shipment methods. Marketing strategies could influence the demand as well as changing the backlogging costs. The closed form expression (15) enables a first cut estimate of the cost impact of any changes in the parameters. Our analysis suggests, for example, that management should focus on reducing the lead time at the upstream stages while reducing the holding cost at the downstream stages. If process re-sequencing is an option, the lowest value added processes with the longest processing times should be carried out sooner rather than later. In addition, the bound can provide an estimate of the decrease in cost that results from advanced demand information as explained in [7] and increases in customer lead times as explained in [9]. The model can also be used to study the benefits of outsourcing by truncating the supply chain using contractual costs instead. In a recent study, Lutze and Özer [10] use similar closed form solutions to design a mutually beneficial contract, referred to as a promised lead time contract, that trades of the value of advanced demand information to the seller and the cost of customer lead time to the buyer in a two level multi period supply chain.

\section{Numerical Study}

Here, we report the performance of our heuristic and of the distribution-free bound. We compare the exact solution to the newsvendor heuristics and report the percentage error $\epsilon^{i} \%=\frac{c_{J}\left(s_{J}^{i}\right)-c_{J}\left(s_{J}^{*}\right)}{c_{J}\left(s_{J}^{*}\right)}$ for $i=\{S S, G O\}$. Shang and Song (2003) use $s_{j}^{S S} \equiv \frac{s_{j}^{*}\left(h_{j}\right)+s_{j}^{*}\left(h_{1}\right)}{2}$ and truncate this average when $p \leq 39$ and round it otherwise. We use $s_{j}^{G O} \equiv s_{j}^{*}\left(h_{j}^{G O}\right)$. By considering a larger set of experiments, we complement the numerical study in Shang and Song (2002). In particular, our numerical study includes unequal leadtimes. To manage the series system, we use an echelon base stock policy with echelon base stock levels $s_{j}^{G O}$ for all $j$. The approximate cost is given by $G_{J}\left(s_{J}^{G O}\right)+\sum_{i=1}^{J} h_{i+1} E D_{i}$. Shang and Song (2003) approximate the optimal cost by $G_{J}\left(s_{J}^{*}\left(h_{J}\right)+\sum_{i=1}^{J} h_{i+1} E D_{i}\right.$ instead of the average since the lower bounds become looser as the number of stages in the system increases. We study two sets of experiments: constant leadtime set and the randomized parameters set.

The first set of experiments is similar to that of Gallego and Zipkin [8] and Shang and Song [13]. The holding cost and the lead times are normalized so $h_{1}=1$ and $L[1, J]=1$. We consider $J \in$ $\{2,4,8,16,32,64\} ; \lambda \in\{16,64\}$; and $p \in\{9,39\}$ (corresponding to fill rates of $90 \%, 97.5 \%$ ). Within this group we consider linear holding-cost form $\left(h_{j}^{e}=1 / J\right)$; affine holding cost form $\left(h^{e}[1, j]=\right.$ $\alpha+(1-\alpha) j / J$ with $\alpha=0.25$ and 0.75$)$; kink holding cost form $\left(h_{j}^{e}=(1-\alpha) / J\right.$ for $j \geq J / 2+1$ and $h_{j}^{e}=(1+\alpha) / J$ for $j<J / 2+1$ with $\alpha=0.25$ and 0.75$)$ and jump holding cost form $\left(h_{j}^{e}=\alpha+(1-\alpha) / J\right.$ for $j=N / 2$ and $h_{j}^{e}=(1-\alpha) / J$ for $j \neq J / 2$ with $\alpha=0.25$ and 0.75$)$. Notice that Shang and Song (2002) consider only the case for $\lambda=64$ and $p=39$. We report the results in Table 1 .

Out of 108 problem instances, in 24 cases the $s^{G O}$ and in 20 cases the $s^{S S}$ heuristic resulted in 
the same solution as the recursive optimization. The $s^{G O}$ (resp., $s^{S S}$ ) heuristic outperforms in 48 (resp., 44) cases and they tie in 17 cases. The average error for $s^{G O}$ (resp., $s^{S S}$ ) heuristic is $0.195 \%$ (resp., $0.385 \%$ ), while the maximum error is $3.68 \%$ and $1.24 \%$ for the GO and the SS heuristics respectively. The quality of the heuristics seems to deteriorate as the number of stages in the system exceeds 32. The SS heuristic seems to perform better for the jump holding cost case, while the GO heuristic tends to dominate in the other cases.

The second set of experiments allow for unequal leadtimes. It is here that we expect the GO heuristic to perform better. To cover a wider range of problem instances we generate the leadtimes and holding costs from uniform distributions. In particular, we use the following set of parameters:

$$
\begin{gathered}
h_{j}^{e} \in\{\operatorname{Unif}(0,1), \operatorname{Unif}(0,5), \operatorname{Unif}(1,10)\}, \\
L_{j} \in\{\operatorname{Unif}(1,2), \operatorname{Unif}(1,10), \operatorname{Unif}(1,40)\}, \\
J \in\{2,4,8,16,32\} \quad b \in\{1,9,39,49\} \quad \lambda \in\{1,3,6\} .
\end{gathered}
$$

We consider 25 combinations, taken at random, from the above parameters. For each subgroup we generate 40 problem instances and calculate the worst case as well as the average performances. We present some of the problem instances in Table 2.

Out of 1000 problem instances, in 188 cases the $s^{G O}$ heuristic and in 133 cases the $s^{S S}$ heuristic resulted in the exact solution. In 849 cases the error term for $s^{G O}$ heuristic is smaller or equal to that of $s^{S S}$ heuristic. The average error for the $s^{G O}$ (resp., $s^{S S}$ ) heuristic is $0.23 \%$ (resp., $0.83 \%$ ). We observe that as the variance of the leadtimes across stages increases the average error term for $s^{G O}$ decreases (the average error for $L_{j} \sim \operatorname{Unif}(1,10)$ is $0.14 \%$ whereas it is $0.39 \%$ for $L_{j} \sim \operatorname{Unif}(1,2)$ ). Similarly the $s^{G O}$ heuristic performs even better as the variance of echelon costs across stages in a series system increases. In the second half of Table 2 we present the ten worst cases that we encountered. The maximum worst case we observed was $3.62 \%$ for the $s^{G O}$ heuristic and $4.36 \%$ for the $s^{S S}$ heuristic.

In light of our numerical observations we suggest the $s^{G O}$ heuristics for a series system with a small number of stages $(J \leq 32)$ especially for systems with high variability in leadtimes and echelon holding costs across stages. More caution should be used for system with a large number of stages and for systems with jump holding costs.

We have also performed a numerical study comparing the actual cost to the distribution-free bound by performing simple linear regressions of the bound to the actual cost by fixing all but one of the parameters. The results of the regressions can be found in Tables 3 and 4 where we report the coefficients of determination $R^{2}$ for the different regressions. Notice that in all cases $R^{2}$ is close to 1. This observation suggests that the bound can safely be used to investigate the impact of process and design changes on the cost of managing a series system.

The simple newsvendor heuristic and the bound enable a manager to quantify the impact of re-sequencing a process. Consider, for example, a four stage series system where $h_{1}=L[1, J]=1$, $p=1$ and $\lambda=16$. We now compare two systems with different configurations of leadtimes. The first system has leadtimes $(0.1,0.1,0.1,0.7)$ and the second has leadtimes $(0.7,0.1,0.1,0.1)$. The 
costs based on the distribution free bound (resp., recursive optimization) are 13.29 (resp., 12.77) for the first system and 5 (resp.,4.93) for the second system. The distribution free bound predicts a cost reduction of $62.4 \%$ while the actual cost reduction based on recursive optimization is $61.39 \%$. This indicates that the distribution free bound enables a quick, yet accurate, what if analysis. In this case, we observe that postponing the shortest and the most expensive processes to a later stage can significantly reduce inventory related costs.

We explored using different weighting structures to approximate holding cost structure for the newsvendor heuristic. In particular, we used $\sum_{k=1}^{j} \frac{L_{k}^{\alpha}}{\sum_{i=1}^{j} L_{i}^{\alpha}} h_{k}$ for different $\alpha \in[0,1]$. We were unable to identify an $\alpha$ that consistently results in lower error terms than $\alpha=1$. In addition, we also used the holding cost structure $H_{j}=\sum_{k=1}^{j-1} h_{k+1} \frac{L_{k}}{\sum_{i=1}^{j-1} L_{i}}$ for $j \geq 2$, and $H_{1}=h_{1}$ that equates the pipeline holding cost under the approximation to the real pipeline cost. This holding cost structure did not perform better than the original proposed holding cost structure. To further investigate what other weighting strategies could work, we have also calculated the implied holding costs $h_{j}^{i m}$ for some problem instances. These holding cost when used in the newsvendor problem of Equation (13) yield the optimal echelon base stock levels $s_{j}^{*}$ obtained through the exact algorithm. In other words we set $h_{j}^{\min } \equiv \min \left\{h \in \mathcal{R}_{+}: s_{j}^{*}(h)=s_{j}^{*}\right\}$ and $h_{j}^{\max } \equiv \min \left\{h \in \mathcal{R}_{+}: s_{j}^{*}(h)=s_{j}^{*}-1\right\}$. Note that using an implied holding cost $h_{j}^{i m} \in\left[h_{j}^{\min }, h_{j}^{\max }\right.$ ) in Equation (13) yields the optimal echelon base stock level. In Table 5 we provide some examples. Due to the robustness of newsvendor cost the range for possible implied holding cost is large. The holding $\operatorname{costs} h_{j}^{G O}$ fall into this range for a large set of problem instances.

\section{Conclusion}

The results of this letter can also be applied to assembly systems by following the ideas in Rosling [12]. For distribution systems, the heuristic can be applied after using the decomposition principles in Gallego, Özer and Zipkin [6]. Our formulation can also be used to model whether or not it is beneficial to outsource an upstream portion of the supply chain by comparing the cost of the original supply chain and the cost of the truncated supply chain with the unit cost from the new source of supply.

\section{References}

[1] Axsater, S. 2003. Serial and Distribution Inventory Systems. Chapter 10 in Vol 11 Handbooks in ORMS, Supply Chain Manangement: Design, Coordination and Operation. Eds. A.G. de Kok and S.C. Graves. Elsevier, The Netherlands.

[2] Chen, F. and Y. Zheng. 1994. Lower Bounds for Multi-echelong Stochastic Inventory Systems. Management Science 40, 1426-1443. 
[3] Clark, A. and H. Scarf. 1960. Optimal Policies for a Multi Echelon Inventory Problem. Management Science 6 475-490.

[4] Federgruen, A. and P. Zipkin. 1984. Computational Issues in an Infinite Horizon, Multiechelon Inventory Model. Operations Research. 32, 818-836.

[5] Gallego G. and I. Moon. 1993. The Distribution Free Newsboy Problem: Review and Extensions. Journal of Operational Research Society. 44, 825-834.

[6] Gallego G, Ö. Özer, and P. Zipkin. 1999. Bounds, Heuristics and Approximations for Distribution Systems. Working Paper.

[7] Gallego, G. and Ö. Özer. 2003. Optimal Replenishment Policies for Multi-Echelon Inventory Problems under Advance Demand Information. MESOM, 5, 157-175.

[8] Gallego G. and P. Zipkin. 1999. Stock Positioning and Performance Estimation in Serial Production-Transportation Systems. MESSOM 1, 77-87.

[9] Hariharan, R. and P. Zipkin. 1995. Customer Order Information, Lead Times, and Inventories. Management Science. 41, 1599-1607.

[10] Lutze, H. and Ö. Özer. 2004. Promised Leadtime Contracts and Renegotiation Incentives Under Asymmetric Information. Working Paper. Stanford University.

[11] Scarf, H. 1958. A Min-Max Solution of an Inventory Problem. Ch. 12 in Studies in The Mathematical Theory of Inventory and Production, Stanford Univ. Press.

[12] Rosling, K. 1989. Optimal Inventory Policies for Assembly Systems Under Random Demands. Operations Research 37, 565-579.

[13] Shang H. K. and J. Song. 2003. Newsvendor Bounds and Heuristics for Optimal Policies in Serial Supply Chains. Management Science 49, 618-638.

[14] Zipkin P. 2000. Foundations of Inventory Management. The Irwin McGraw Hill Series. 
Table 1: Comparison of Optimal and Heuristic Policy

\begin{tabular}{|c|c|c|c|c|c|c|c|c|c|c|c|c|}
\hline \multicolumn{13}{|c|}{$(\lambda=16, p=39)$} \\
\hline $\mathrm{N}$ & Form & $c\left(s_{J}^{S S}\right)$ & $c\left(s_{J}^{G O}\right)$ & $c\left(s_{J}^{*}\right)$ & $\epsilon^{S S} \%$ & $\epsilon^{G O_{\%}}$ & Form & $c\left(s_{J}^{S S}\right)$ & $c\left(s_{J}^{G O}\right)$ & $c\left(s_{J}^{*}\right)$ & $\epsilon^{S S} \%$ & $\epsilon^{G O} \%$ \\
\hline 64 & Kink & 18.066 & 17.957 & 17.775 & 1.637 & 1.024 & Kink & 20.839 & 20.471 & 20.381 & 2.247 & 0.442 \\
\hline 32 & $\alpha=0.25$ & 17.860 & 17.793 & 17.668 & 1.087 & 0.707 & $\alpha=0.75$ & 20.543 & 20.326 & 20.266 & 1.367 & 0.296 \\
\hline 16 & & 17.557 & 17.527 & 17.461 & 0.550 & 0.378 & & 20.162 & 20.040 & 20.034 & 0.639 & 0.030 \\
\hline 8 & & 17.099 & 17.075 & 17.063 & 0.211 & 0.070 & & 19.675 & 19.608 & 19.604 & 0.362 & 0.020 \\
\hline 4 & & 16.246 & 16.244 & 16.244 & 0.012 & 0.000 & & 18.759 & 18.726 & 18.726 & 0.176 & 0.000 \\
\hline 2 & & 14.617 & 14.617 & 14.617 & 0.000 & 0.000 & & 17.063 & 16.999 & 16.999 & 0.376 & 0.000 \\
\hline 64 & Affine & 19.119 & 19.074 & 18.955 & 0.865 & 0.628 & Affine & 23.699 & 23.722 & 23.656 & 0.182 & 0.279 \\
\hline 32 & $\alpha=0.25$ & 18.957 & 18.897 & 18.814 & 0.760 & 0.441 & $\alpha=0.75$ & 23.481 & 23.495 & 23.440 & 0.175 & 0.235 \\
\hline 16 & & 18.532 & 18.579 & 18.525 & 0.038 & 0.291 & & 23.053 & 23.066 & 23.012 & 0.178 & 0.235 \\
\hline 8 & & 17.985 & 18.000 & 17.975 & 0.056 & 0.139 & & 22.202 & 22.163 & 22.159 & 0.194 & 0.018 \\
\hline 4 & & 16.843 & 16.843 & 16.843 & 0.000 & 0.000 & & 20.494 & 20.442 & 20.440 & 0.264 & 0.010 \\
\hline 2 & & 14.617 & 14.617 & 14.617 & 0.000 & 0.000 & & 17.063 & 16.999 & 16.999 & 0.376 & 0.000 \\
\hline 64 & Jump & 16.536 & 16.598 & 16.280 & 1.572 & 1.953 & Linear & 16.621 & 16.612 & 16.409 & 1.292 & 1.237 \\
\hline 32 & $\alpha=0.25$ & 16.301 & 16.375 & 16.142 & 0.985 & 1.443 & & 16.428 & 16.427 & 16.296 & 0.810 & 0.804 \\
\hline 16 & & 15.977 & 16.137 & 15.861 & 0.731 & 1.740 & & 16.174 & 16.238 & 16.094 & 0.497 & 0.895 \\
\hline 8 & & 15.339 & 15.478 & 15.317 & 0.144 & 1.051 & & 15.704 & 15.759 & 15.703 & 0.006 & 0.357 \\
\hline 4 & & 14.204 & 14.306 & 14.204 & 0.000 & 0.718 & & 14.956 & 14.975 & 14.954 & 0.013 & 0.140 \\
\hline 2 & & 12.011 & 12.011 & 12.011 & 0.000 & 0.000 & & 13.314 & 13.314 & 13.314 & 0.000 & 0.000 \\
\hline \multicolumn{13}{|c|}{$(\lambda=16, p=9)$} \\
\hline 64 & Kink & 15.863 & 15.614 & 15.565 & 1.915 & 0.315 & Kink & 18.390 & 17.953 & 17.938 & 2.520 & 0.084 \\
\hline 32 & $\alpha=0.25$ & 15.602 & 15.491 & 15.450 & 0.984 & 0.265 & $\alpha=0.75$ & 18.093 & 17.819 & 17.816 & 1.555 & 0.017 \\
\hline 16 & & 15.265 & 15.248 & 15.239 & 0.171 & 0.059 & & 17.710 & 17.573 & 17.572 & 0.785 & 0.006 \\
\hline 8 & & 14.805 & 14.776 & 14.776 & 0.196 & 0.000 & & 17.108 & 17.108 & 17.108 & 0.000 & 0.000 \\
\hline 4 & & 13.922 & 13.891 & 13.891 & 0.223 & 0.000 & & 16.205 & 16.205 & 16.205 & 0.000 & 0.000 \\
\hline 2 & & 12.166 & 12.138 & 12.138 & 0.231 & 0.000 & & 14.332 & 14.332 & 14.332 & 0.000 & 0.000 \\
\hline 64 & Affine & 16.705 & 16.713 & 16.676 & 0.174 & 0.222 & Affine & 21.053 & 21.123 & 21.047 & 0.029 & 0.361 \\
\hline 32 & $\alpha=0.25$ & 16.540 & 16.553 & 16.529 & 0.067 & 0.145 & $\alpha=0.75$ & 20.830 & 20.902 & 20.830 & 0.000 & 0.346 \\
\hline 16 & & 16.250 & 16.241 & 16.231 & 0.117 & 0.062 & & 20.402 & 20.400 & 20.398 & 0.020 & 0.010 \\
\hline 8 & & 15.655 & 15.649 & 15.643 & 0.077 & 0.038 & & 19.533 & 19.533 & 19.533 & 0.000 & 0.000 \\
\hline 4 & & 14.517 & 14.489 & 14.483 & 0.235 & 0.041 & & 17.804 & 17.804 & 17.804 & 0.000 & 0.000 \\
\hline 2 & & 12.166 & 12.138 & 12.138 & 0.231 & 0.000 & & 14.332 & 14.332 & 14.332 & 0.000 & 0.000 \\
\hline 64 & Jump & 14.358 & 14.336 & 14.196 & 1.141 & 0.986 & Linear & 14.506 & 14.379 & 14.317 & 1.320 & 0.433 \\
\hline 32 & $\alpha=0.25$ & 14.115 & 14.145 & 14.051 & 0.455 & 0.669 & & 14.304 & 14.231 & 14.201 & 0.725 & 0.211 \\
\hline 16 & & 13.806 & 13.840 & 13.755 & 0.371 & 0.618 & & 14.021 & 14.007 & 13.980 & 0.293 & 0.193 \\
\hline 8 & & 13.187 & 13.228 & 13.178 & 0.068 & 0.379 & & 13.574 & 13.580 & 13.558 & 0.118 & 0.162 \\
\hline 4 & & 12.031 & 12.061 & 12.031 & 0.000 & 0.249 & & 12.688 & 12.688 & 12.688 & 0.000 & 0.000 \\
\hline 2 & & 9.710 & 9.710 & 9.710 & 0.000 & 0.000 & & 10.924 & 10.924 & 10.924 & 0.000 & 0.000 \\
\hline \multicolumn{13}{|c|}{$(\lambda=64, p=39)$} \\
\hline 64 & Kink & 52.742 & 52.667 & 52.352 & 0.745 & 0.602 & Kink & 62.378 & 61.848 & 61.622 & 1.227 & 0.367 \\
\hline 32 & alpha $=0.25$ & 52.086 & 52.130 & 51.903 & 0.353 & 0.437 & alpha $=0.75$ & 61.610 & 61.313 & 61.157 & 0.741 & 0.255 \\
\hline 16 & & 51.099 & 51.140 & 51.011 & 0.173 & 0.253 & & 60.551 & 60.327 & 60.226 & 0.540 & 0.168 \\
\hline 8 & & 49.259 & 49.259 & 49.223 & 0.073 & 0.073 & & 58.484 & 58.425 & 58.381 & 0.176 & 0.075 \\
\hline 4 & & 45.660 & 45.721 & 45.660 & 0.000 & 0.134 & & 54.773 & 54.675 & 54.675 & 0.179 & 0.000 \\
\hline 2 & & 38.457 & 38.457 & 38.457 & 0.000 & 0.000 & & 47.314 & 47.267 & 47.267 & 0.099 & 0.000 \\
\hline 64 & Affine & 56.877 & 56.959 & 56.707 & 0.300 & 0.444 & Affine & 74.105 & 74.165 & 74.085 & 0.027 & 0.108 \\
\hline 32 & alpha $=0.25$ & 56.140 & 56.300 & 56.120 & 0.036 & 0.321 & alpha $=0.75$ & 73.240 & 73.279 & 73.222 & 0.025 & 0.078 \\
\hline 16 & & 54.966 & 55.053 & 54.954 & 0.022 & 0.180 & & 71.514 & 71.540 & 71.495 & 0.027 & 0.063 \\
\hline 8 & & 52.615 & 52.684 & 52.609 & 0.011 & 0.143 & & 68.065 & 68.085 & 68.042 & 0.034 & 0.063 \\
\hline 4 & & 47.931 & 47.925 & 47.921 & 0.021 & 0.008 & & 61.160 & 61.128 & 61.128 & 0.052 & 0.000 \\
\hline 2 & & 38.475 & 38.457 & 38.457 & 0.047 & 0.000 & & 47.314 & 47.267 & 47.267 & 0.099 & 0.000 \\
\hline 64 & Jump & 46.107 & 47.742 & 46.075 & 0.069 & 3.618 & Linear & 47.859 & 48.045 & 47.590 & 0.565 & 0.956 \\
\hline 32 & alpha $=0.75$ & 45.231 & 46.640 & 45.217 & 0.031 & 3.147 & & 47.289 & 47.518 & 47.151 & 0.293 & 0.778 \\
\hline 16 & & 43.518 & 44.647 & 43.500 & 0.041 & 2.637 & & 46.335 & 46.487 & 46.265 & 0.151 & 0.480 \\
\hline 8 & & 40.080 & 40.892 & 40.069 & 0.027 & 2.054 & & 44.555 & 44.658 & 44.529 & 0.058 & 0.290 \\
\hline 4 & & 33.204 & 33.864 & 33.188 & 0.048 & 2.037 & & 41.015 & 41.034 & 41.015 & 0.000 & 0.046 \\
\hline 2 & & 19.389 & 19.655 & 19.370 & 0.098 & 1.471 & & 33.916 & 33.916 & 33.916 & 0.000 & 0.000 \\
\hline
\end{tabular}


Table 2: Problem instances for $J=4$.

\begin{tabular}{|c|c|c|c|c|c|c|c|c|c|}
\hline$\left(L_{4}, L_{3}, L_{2}, L_{1}\right)$ & $\left(h_{4}^{e}, h_{3}^{e}, h_{2}^{e}, h_{1}^{e}\right)$ & $s^{S S}$ & $c_{4}\left(s_{4}^{S S}\right)$ & $s^{G O}$ & $c_{4}\left(s_{4}^{G O}\right)$ & $\mathbf{s}$ & $c_{4}\left(s_{4}^{*}\right)$ & $\epsilon^{S S} \%$ & $\epsilon^{G O_{\%}}$ \\
\hline \multicolumn{10}{|l|}{$p=49, \lambda=1$} \\
\hline$(1.698,1.067,1.274,1.676)$ & $(9.928,2.889,4.290,1.521)$ & $(8,7,6,5)$ & 113.143 & $(7,7,6,5)$ & 110.650 & $(7,7,5,5)$ & 110.606 & $2.29 \%$ & $0.04 \%$ \\
\hline$(1.241,1.200,1.442,1.939)$ & $(5.818,7.261,2.996,6.118)$ & $(8,7,6,4)$ & 119.258 & $(7,6,6,4)$ & 117.140 & $(7,6,6,4)$ & 117.140 & $1.81 \%$ & $0 \%$ \\
\hline$(1.412,1.749,1.071,1.798)$ & $(9.804,5.981,4.367,1.712)$ & $(8,8,6,5)$ & 135.000 & $(8,7,5,5)$ & 134.123 & $(7,7,5,5)$ & 132.365 & $2.04 \%$ & $1.33 \%$ \\
\hline$(1.545,1.662,1.503,1.700)$ & $(5.982,4.047,4.553,2.421)$ & $(9,8,6,4)$ & 105.408 & $(9,7,6,4)$ & 105.094 & $(8,7,6,4)$ & 103.954 & $1.40 \%$ & $1.10 \%$ \\
\hline$(1.077,1.186,1.291,1.082)$ & $(6.992,7.452,6.848,6.558)$ & $(6,6,4,3)$ & 112.003 & $(6,5,4,3)$ & 110.826 & $(6,5,4,3)$ & 110.826 & $1.06 \%$ & $0 \%$ \\
\hline$(1.840,1.019,1.772,1.663)$ & $(9.896,2.546,2.596,8.907)$ & $(8,7,6,3)$ & 120.961 & $(8,7,6,3)$ & 120.961 & $(8,7,6,3)$ & 120.961 & $0 \%$ & $0 \%$ \\
\hline$(1.969,1.575,1.250,1.872)$ & $(2.681,8.524,2.728,5.110)$ & $(10,7,6,4)$ & 101.835 & $(9,7,6,4)$ & 100.681 & $(9,6,6,4)$ & 99.852 & $1.99 \%$ & $0.83 \%$ \\
\hline$(1.434,1.382,1.818,1.403)$ & $(3.391,5.041,2.926,6.195)$ & $(9,7,6,3)$ & 87.307 & $(8,7,6,3)$ & 86.457 & $(8,7,6,3)$ & 86.457 & $0.98 \%$ & $0 \%$ \\
\hline$(1.204,1.600,1.800,1.885)$ & $(5.879,4.950,8.401,9.215)$ & $(9,8,6,4)$ & 141.850 & $(8,7,5,4)$ & 136.538 & $(8,7,5,4)$ & 136.530 & $3.89 \%$ & $0 \%$ \\
\hline$(1.032,1.664,1.813,1.687)$ & $(3.185,2.163,1.310,5.019)$ & $(9,9,7,4)$ & 65.950 & $(9,8,7,4)$ & 65.937 & $(9,8,7,4)$ & 65.937 & $0.02 \%$ & $0 \%$ \\
\hline \multicolumn{10}{|c|}{ Some of the observed worst cases for both heuristics } \\
\hline \multicolumn{10}{|l|}{$p=49, \lambda=1$} \\
\hline$(1.222,1.765,1.938,1.732)$ & $(7.719,9.942,4.913,9.118)$ & $(9,8,6,3)$ & 172.428 & $(8,7,6,3)$ & 165.232 & $(8,7,6,3)$ & 165.232 & $4.355 \%$ & $0 \%$ \\
\hline$(1.862,1.057,1.462,1.842)$ & $(7.542,1.643,3.786,5.147)$ & $(9,8,6,4)$ & 102.465 & $(8,7,6,4)$ & 99.765 & $(8,7,6,4)$ & 99.765 & $2.707 \%$ & $0 \%$ \\
\hline$(1.561,1.254,1.644,1.979)$ & $(3.155,8.428,9.658,4.546)$ & $(9,7,6,4)$ & 135.289 & $(8,6,5,4)$ & 131.429 & $(8,6,5,4)$ & 131.429 & $2.937 \%$ & $0 \%$ \\
\hline$(1.599,1.422,1.013,1.103)$ & $(6.410,3.175,2.005,1.374)$ & $(8,7,5,4)$ & 75.141 & $(7,6,5,4)$ & 72.836 & $(7,6,5,4)$ & 72.836 & $3.165 \%$ & $0 \%$ \\
\hline$(1.159,1.583,1.929,1.404)$ & $(8.716,1.984,3.435,3.370)$ & $(9,8,6,4)$ & 112.422 & $(8,8,6,4)$ & 108.227 & $(8,8,6,4)$ & 108.227 & $3.876 \%$ & $0 \%$ \\
\hline$(1.412,1.749,1.071,1.798)$ & $(9.804,5.981,4.367,1.712)$ & $(8,8,6,5)$ & 135.06 & $(8,7,5,5)$ & 134.123 & $(7,7,5,5)$ & 132.365 & $2.04 \%$ & $1.33 \%$ \\
\hline \multicolumn{10}{|l|}{$p=1, \lambda=1$} \\
\hline$(1.949,1.921,1.550,1.346)$ & $(0.472,0.375,0.847,0.317)$ & $(7,6,3,3)$ & 7.328 & $(6,5,3,3)$ & 7.126 & $(6,5,3,3)$ & 7.126 & $2.823 \%$ & $0 \%$ \\
\hline$(1.009,1.919,1.276,1.273)$ & $(0.588,0.691,0.838,0.726)$ & $(5,4,3,2)$ & 8.312 & $(5,4,3,2)$ & 8.312 & $(4,4,3,2)$ & 8.138 & $2.133 \%$ & $2.133 \%$ \\
\hline$(1.693,1.303,1.427,1.070)$ & $(0.967,0.683,0.153,0.877)$ & $(4,4,4,2)$ & 8.177 & $(5,4,4,2)$ & 8.352 & $(4,4,4,2)$ & 8.177 & $0 \%$ & $2.137 \%$ \\
\hline$(1.545,1.448,1.409,1.299)$ & $(0.466,0.501,0.153,0.323)$ & $(6,5,4,3)$ & 5.767 & $(6,5,5,3)$ & 5.766 & $(5,5,5,3)$ & 5.662 & $1.866 \%$ & $1.845 \%$ \\
\hline
\end{tabular}

Table 3: Performance of the Distribution Free Bound for factors $N, b$, and $\lambda$.

\begin{tabular}{|rrr|rrr|rrr|}
\hline$p=10$ & $\lambda=16$ & $R^{2}=$ & $J=4$ & $\lambda=16$ & $R^{2}=$ & $J=4$ & $p=10$ & $R^{2}=$ \\
$h_{i}^{e}=0.25$ & $L_{i}=0.25$ & $99.99 \%$ & $h_{i}^{e}=0.25$ & $L_{i}=0.25$ & $96.63 \%$ & $h_{i}^{e}=0.25$ & $L_{i}=0.25$ & $99.97 \%$ \\
\hline $\mathrm{J}$ & $\mathrm{DFB}$ & $c\left(s_{J}^{*}\right)$ & $\mathrm{p}$ & $\mathrm{DFB}$ & $c\left(s_{4}^{*}\right)$ & $\lambda$ & $\mathrm{DFB}$ & $c\left(s_{4}^{*}\right)$ \\
\hline 2 & 6.48 & 4.03 & 1 & 9.16 & 9.05 & 2 & 4.29 & 3.43 \\
3 & 10.75 & 7.87 & 2 & 10.47 & 10.17 & 4 & 6.50 & 5.14 \\
4 & 16.00 & 12.87 & 4 & 12.33 & 11.34 & 6 & 8.37 & 6.62 \\
5 & 22.25 & 18.95 & 6 & 13.75 & 12.02 & 8 & 10.07 & 7.98 \\
6 & 29.49 & 26.10 & 8 & 14.94 & 12.48 & 10 & 11.66 & 9.27 \\
7 & 37.73 & 34.32 & 10 & 16.00 & 12.87 & 12 & 13.16 & 10.50 \\
8 & 46.97 & 43.59 & 12 & 16.95 & 13.15 & 14 & 14.60 & 11.69 \\
10 & 68.45 & 65.25 & 14 & 17.83 & 13.38 & 16 & 16.00 & 12.87 \\
12 & 93.93 & 90.98 & 16 & 18.65 & 13.59 & 20 & 18.68 & 15.11 \\
14 & 123.40 & 120.77 & 18 & 19.42 & 13.77 & 30 & 24.94 & 20.47 \\
16 & 156.87 & 154.60 & 20 & 20.14 & 13.94 & 32 & 26.14 & 21.50 \\
\hline
\end{tabular}

Table 4: Performance of Distribution Free Bound wrt factors $h_{4}, h_{1}, L_{4}$, and $L_{1}$ When $J=4$

\begin{tabular}{|rrr|rrr|rrrrr|r|r|}
\hline$p=10$ & $\lambda=16$ & $R^{2}=$ & $p=10$ & $\lambda=16$ & $R^{2}=$ & $p=10$ & $\lambda=16$ & $R^{2}=$ & $p=10$ & $\lambda=16$ & $R^{2}=$ \\
$h_{i}^{e}=0.25$ & $L_{i}=0.25$ & $99.99 \%$ & $h_{i}^{e}=0.25$ & $L_{i}=0.25$ & $99.07 \%$ & $h_{i}^{e}=0.25$ & $L_{i}=0.25$ & $99.93 \%$ & $h_{i}^{e}=0.25$ & $L_{i}=0.25$ & $99.99 \%$ \\
$h_{4}^{e}$ & $\mathrm{DFB}$ & $c\left(s_{4}^{*}\right)$ & $h_{1}^{e}$ & $\mathrm{DFB}$ & $c\left(s_{4}^{*}\right)$ & $L_{4}$ & $\mathrm{DFB}$ & $c\left(s_{4}^{*}\right)$ & $L_{1}$ & $\mathrm{DFB}$ & $c\left(s_{4}^{*}\right)$ \\
\hline 1 & 29.83 & 26.07 & 1 & 17.40 & 15.12 & 1 & 17.40 & 13.81 & 1 \\
2 & 46.49 & 42.20 & 2 & 19.04 & 17.35 & 29.83 & 24.59 \\
3 & 62.24 & 57.61 & 3 & 20.49 & 19.25 & 3 & 19.04 & 14.68 & 2 & 46.49 & 39.18 \\
4 & 77.46 & 72.43 & 4 & 21.81 & 20.55 & 4 & 21.81 & 15.95 & 3 & 62.23 & 53.23 \\
5 & 92.33 & 86.88 & 5 & 23.03 & 21.83 & 5 & 23.03 & 16.49 & 77.45 & 67.00 \\
6 & 106.94 & 101.04 & 6 & 24.16 & 23.10 & 6 & 24.17 & 16.96 & 6 & 92.33 & 80.60 \\
7 & 121.35 & 115.17 & 7 & 25.23 & 24.02 & 7 & 25.24 & 17.41 & 7 & 106.93 & 94.06 \\
8 & 135.61 & 128.75 & 8 & 26.25 & 24.74 & 8 & 26.25 & 17.82 & 8 & 135.61 & 120.66 \\
9 & 149.73 & 142.34 & 9 & 27.22 & 25.45 & 9 & 27.21 & 18.22 & 9 & 149.72 & 133.85 \\
10 & 163.74 & 155.93 & 10 & 28.14 & 26.16 & 10 & 28.14 & 18.59 & 10 & 163.74 & 146.98 \\
\hline
\end{tabular}


Table 5: Implied Holding Costs When $J=4$

\begin{tabular}{|c|c|c|c|c|}
\hline$\left(L_{4}, L_{3}, L_{2}, L_{1}\right)$ & $\left(h_{4}, h_{3}, h_{2}, h_{1}\right)$ & $\left(h_{4}^{G O}, h_{3}^{G O}, h_{2}^{G O}, h_{1}^{G O}\right)$ & $\left(h_{4}^{\text {min }}, h_{3}^{\text {min }}, h_{2}^{\text {min }}, h_{1}^{\text {min }}\right)$ & $\left(h_{4}^{\max }, h_{3}^{\max }, h_{2}^{\max }, h_{1}^{\max }\right)$ \\
\hline \multicolumn{5}{|l|}{$p=9, \lambda=1$} \\
\hline$(7.279,1.606,3.467,7.087)$ & $(9.928,12.817,17.107,18.628)$ & $(14.619,17.427,18.128,18.628)$ & $(12.154,15.991,16.761,17.909)$ & $(17.495,19.769,19.586,18.805)$ \\
\hline$(7.570,6.158,8.306,3.782)$ & $(2.134,6.763,16.100,18.691)$ & $(10.157,13.486,16.911,18.691)$ & $(8.372,13.603,15.611,18.347)$ & $(11.608,18.867,20.898,21.495)$ \\
\hline$(3.265,8.386,5.579,3.418)$ & $(9.892,19.141,21.237,24.797)$ & $(19.181,20.926,22.589,24.797)$ & $(19.271,19.270,21.651,22.701)$ & $(27.310,23.834,23.768,25.026)$ \\
\hline$(6.126,3.384,2.003,3.404)$ & $(7.601,17.398,22.789,25.723)$ & $(15.998,21.850,24.636,25.723)$ & $(12.598,17.641,23.521,24.258)$ & $(19.031,24.803,29.712,26.604)$ \\
\hline$(2.327,9.546,2.274,9.146)$ & $(7.236,10.963,15.802,17.435)$ & $(13.604,14.311,17.110,17.435)$ & $(12.797,13.299,14.888,17.208)$ & $(17.858,16.072,17.567,18.468)$ \\
\hline \multicolumn{5}{|l|}{$p=39, \lambda=1$} \\
\hline$(2.327,9.546,2.274,9.146)$ & $(3.468,4.990,7.129,7.490)$ & $(6.028,6.313,7.418,7.490)$ & $(5.728,5.851,6.839,7.338)$ & $(7.956,6.896,8.024,7.543)$ \\
\hline$(1.210,7.247,3.146,3.558)$ & $(2.820,5.927,10.124,11.574)$ & $(7.875,8.314,10.894,11.574)$ & $(7.314,8.531,10.467,11.560)$ & $(11.030,11.532,14.224,13.502)$ \\
\hline
\end{tabular}

